$\mathbf{E}_{\mathrm{B}}^{\mathrm{n}}$ 1997, la Sociedad para el Estudio de los Recursos Bióticos de Oaxaca (SERBO) inició exploraciones botánicas en la porción este de la costa de Oaxaca, con la finalidad de generar información florística de una de las regiones menos conocidas desde el punto de vista botánico (García-Mendoza y Torres-Colín, 1999) y con alta prioridad para su conservación (Arriaga et al., 2000). Una de las áreas más interesantes de la región explorada es la cuenca del río Zimatán, debido principalmente a su gran variación altitudinal, que abarca desde el nivel del mar hasta casi 3,000 m snm. En consecuencia, en esta cuenca existe una gran diversidad de tipos de vegetación, desde selvas bajas espinosas cercanas al mar, que se desarrollan en condiciones de temperaturas altas y suelos arenosos muy salinos, hasta bosques de pino-encino con influencia de vientos húmedos provenientes del Oceáno Pacífico. Entre estos dos extremos, hacia la porción más húmeda y cubriendo grandes extensiones, se encuentran las selvas medianas subperennifolias, las cuales han sido fuertemente perturbadas debido a la producción de café. Sin embargo, el método empleado para el cultivo de café en esta porción de Oaxaca, en el que se utiliza la sombra de los árboles nativos, ha permitido aproximarnos tanto a la composición florística original, como a la estructura y la diversidad del dosel arbóreo de estas selvas.

Recientemente se han hecho algunos hallazgos botánicos interesantes en esta zona. Uno de ellos es el primer registro para el estado de Oaxaca de Berrya cubensis (Griseb.) M. Gómez (Tiliaceae, figura 1), que se agrega al listado florístico ya publicado para esta área (Salas et al., 2003). Dicho registro representa la distribución más sureña de esta especie en la vertiente mexicana del Pacífico. Téllez (1981) registró a Berrya cubensis por primera vez para la vertiente del Océano Pacífico en la Isla María Madre del estado de Nayarit. La

\title{
BERRYA CUBENSIS (GRISEB.) M. GÓMEZ (TILIACEAE), NUEVO REGISTRO PARA EL estado DE OAXACA, MÉXICO
}

\author{
Silvia H. Salas-Morales \\ Sociedad para el Estudio de los Recursos Bióticos de Oaxaca, A.C., \\ Carretera Internacional Km 7 No. 22, San Sebastián Tutla, C.P. 71246, \\ Oaxaca, México. \\ Correo-e: serbo@prodigy.net.mx
}

especie se ha colectado además en Jalisco, en contra del señalamiento de Pennington y Sarukhán (1998), quienes afirmaron que es una especie exclusiva de la vertiente del Golfo de México, región en la que tiene una distribución más amplia. En México se ha colectado también en Hidalgo, Puebla, Querétaro, San Luis Potosi, Tabasco y Veracruz (según especímenes depositados en los herbarios MEXU y ENCB, (figura 2), y en Colima y Chiapas; su área de distribución se extiende hasta Centro América y el Caribe (Fryxell, 2001).

En Oaxaca Berrya cubensis es un árbol escaso, de 10 a $25 \mathrm{~m}$ de altura. Pierde sus hojas durante la temporada seca del año y crece entremezclada con otros árboles que son utilizados para sombra de café, como Calycophyllum candidissimum, Lonchocarpus molinae, Bernoullia flammea, Cupania dentada, Brosimum alicastrum, Cojoba arborea, Aphananthe monoica y Trophis mexicana, entre otros. Hacia porciones de menor altitud se le encuentra en orilla de arroyos.

Berrya cubensis fue descrita en México con el nombre de Carpoditera amelia, y en varias publicaciones fue consignada utilizando esta denominación; en 2001, P. Fryxell determinó que dicho nombre era un sinónimo de Berrya cubensis.

Esta especie se conoce localmente con el nombre de cortalagua morada y es utilizada para madera. En la cuenca del río Zimatán se desarrolla exclusivamente en las selvas medianas subperennifolias como un elemento escaso, mientras que en la vertiente del Golfo de México al parecer llega a ser una de las especies dominantes (Pennington y Sarukhán, 1998). En México también ha sido colectada en otros tipos de vegetación como selva mediana subcaducifolia, selva alta perennifolia, selva baja caducifolia con encinar, bosque de encino y vegetación secundaria. Los ejemplares provienen de altitudes que varían desde 100 hasta poco más de $800 \mathrm{~m}$, aunque en la cuenca del río Zimatán la menor altitud a la que se le encuentra es $460 \mathrm{~m}$. La época de floración es julio y agosto, y la fructificación ocurre entre noviembre y enero.

\section{Material de referencia}

México. Oaxaca: rancho San Agustín, 1553'19.2”, 9606'07”, 830 m snm, J. Pascual 1091 (árbol de 10 $\mathrm{m}$, fl. morada); $50 \mathrm{~m}$ arriba de la finca El Istmo, 1559'16.8”, 6906'10.3”, $810 \mathrm{~m}$ snm, A. Nava 193 y M. Elorsa (árbol de $20 \mathrm{~m}$, con frutos cafés que giran al caer); $500 \mathrm{~m}$ al este de la finca El Mamey, orilla de río San Lorenzo, 1558'54", 9605'23”, 520 m snm, S. Salas 3947 (árbol de $12 \mathrm{~m}$, fl. rosas); finca El Istmo, 1559'18.8” 


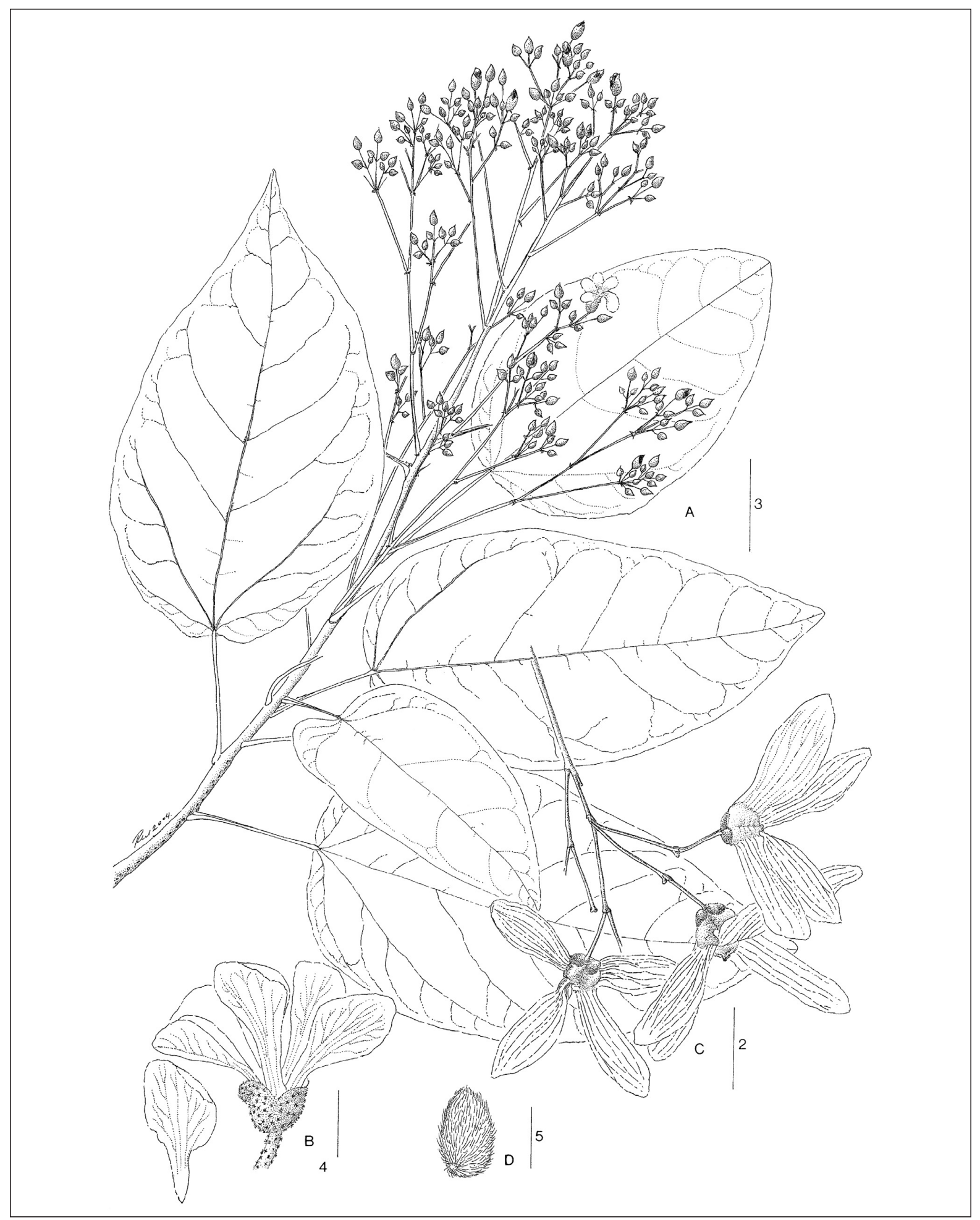

Figura 1. Berrya cubensis. A, rama con inflorescencias $\times 3 \mathrm{~cm}$ (S. Salas 3947); B, flor y pétalo $\times 4 \mathrm{~mm}$ (S. Salas 3947 ); C, frutos $\times 2$ cm (A. Nava 193 y M. Elorza); D, semilla 3 × 5 mm (A. Nava 193 y M. Elorza). 


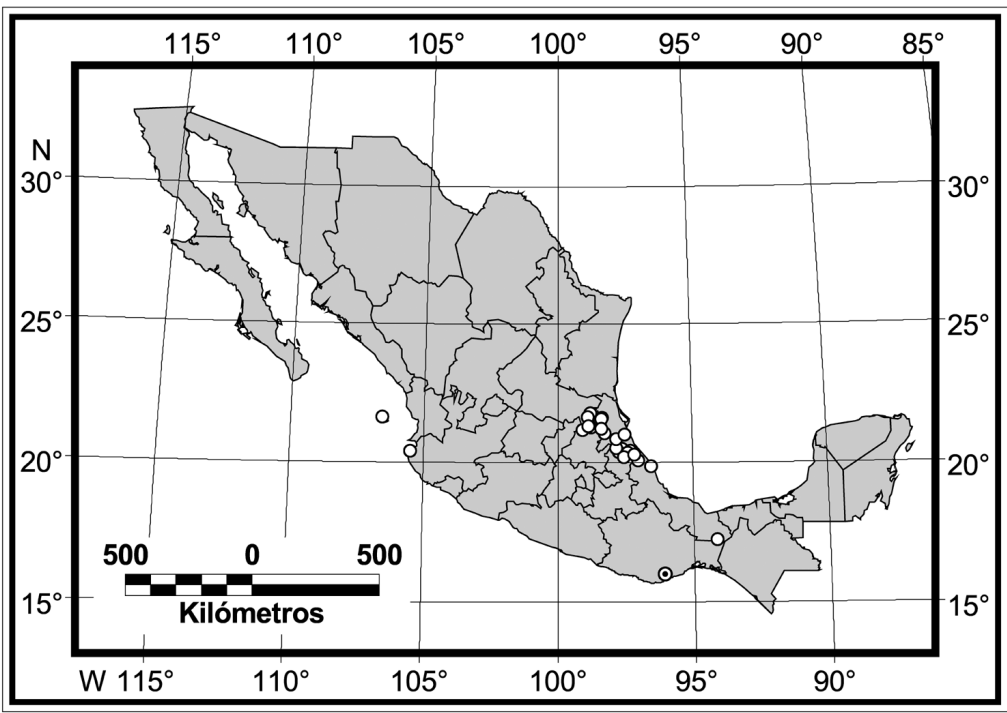

Figura 2. Mapa de distribución de Berrya cubensis en México, según localidades de especímenes depositados en MEXU (círculo) y nueva distribución en Oaxaca (círculo con punto).

9606'05.2”, $810 \mathrm{~m}$ snm, S. Salas 5245 y A. Sánchez (árbol de 20 m, fl. rosas); puente San Lorenzo, $30 \mathrm{~m}$ antes de la desviación a la Finca Montecarlo, orilla de río San Lorenzo, 15'58'39" 9604'36”, 460 m snm, A. Saynes 2210, N. Velásquez, E. Elorsa, E. Martínez, A. Reyes, $M$. Sousa (árbol de $12 \mathrm{~m}$, fl. morada); depositados en los herbarios MEXU, GUIE', OAX e IEB.

\section{Agradecimentos}

Un agradecimiento especial a $\mathrm{T}$. Gregory por su confianza y apoyo económico para llevar a cabo exploraciones botánicas en la cuenca alta del río Zimatán. A. Saynes Vásquez, A. Sánchez Martínez, A. Nava Zafra y J. Pascual Cortés colaboraron en el trabajo de campo. L. Schibli hizo valiosas sugerencias al escrito $y$ elaboró el mapa de distribución. R. Cruz dibujó la ilustración y M. Mora
Jarvio recopiló la información de herbario. Oswaldo Téllez Valdés revisó el escrito e hizo útiles y acertadas sugerencias.

\section{Literatura citada}

Arriaga L., Espinosa J.M., Aguilar C., Martínez E. Gómez L. y Loa E. Coord. 2000. Regiones Terrestres Prioritarias de México. Comisión Nacional para el Conocimiento y Uso de la Biodiversidad, México, D.F.

Fryxell P. 2001. Tiliaceae 1. En: MacVaugh R. Ed. Flora NovoGaliciana. A Descriptive Account of the Vascular Plants of Western Mexico. Vol. 3: Ochnaceae to Loasaceae, pp.71-73, The University of Michigan Press, Ann Arbor, Michigan.

García-Mendoza A. y Torres-Colín R. 1999. Estado actual del conocimiento sobre la flora de Oaxaca. En: VásquezDávila M.A. Ed. Sociedad y Naturaleza de Oaxaca 3: Vegetación y
Flora, pp. 50-86, Instituto Tecnológico Agropecuario de Oaxaca, Oaxaca.

Pennington T.D. y Sarukhán J. 1998. Árboles Tropicales de México. Manual para la Identificación de las Principales Especies. 2a ed., Universidad Nacional Autónoma de México y Fondo de Cultura Económica, México, D.F.

Salas-Morales S.H., Saynes-Vásquez A. y Schibli L. 2003. Flora de la costa de Oaxaca: Lista florística de la región de Zimatán. Boletín de la Sociedad Botánica de México 72:21-58.

Telléz V.O. 1981. Nota sobre la distribución de Carpodiptera ameliae (Tiliaceae) en México. Boletín de la Sociedad Botánica de México 41:166.

Fecha de recepción: 3 de agosto de 2004

Versión corregida: 7 de octubre de 2004

Aceptado:11 de octubre de 2004 